\title{
Epstein-Barr virus-associated inflammatory pseudotumor of the spleen: report of two cases and review of the literature
}

\author{
Lizabeth Rosenbaum • M. Houman Fekrazad • \\ Ian Rabinowitz • Mohammad A. Vasef
}

Received: 4 October 2008 / Accepted: 4 March 2009/Published online: 31 March 2009

(C) Springer-Verlag 2009

\begin{abstract}
We report two rare examples of Epstein-Barr virus (EBV)-associated inflammatory pseudotumor of the spleen. One patient presented with night sweats, abdominal pain, and weight loss and was found to have a splenic mass on CT scan suspected of lymphoma. The splenic mass in second patient was found incidentally at the time of work up for kidney stones. The pathologic examination of these splenectomy specimens showed similar histologic features. However, the spindle cells were composed of EBV-infected follicular dendritic cells in one case whereas the second case lacked significant follicular dendritic cell proliferation and showed only focal EBV-infected cells suggesting that these proliferations are heterogenous in nature.
\end{abstract}

Keywords Splenic inflammatory pseudotumor - EpsteinBarr virus · Follicular dendritic cell

\footnotetext{
L. Rosenbaum $\cdot$ M. A. Vasef

Department of Pathology,

University of New Mexico Health Sciences Center,

Albuquerque, NM, USA

M. H. Fekrazad • I. Rabinowitz

Department of Internal Medicine,

Division of Hematology/Oncology,

University of New Mexico Health Sciences Center,

Albuquerque, NM, USA

M. A. Vasef $(\bowtie)$

Department of Pathology MSC08 4640,

1 University of New Mexico,

Albuquerque, NM 87131, USA

e-mail: mvasef@salud.unm.edu
}

\section{Introduction}

Inflammatory pseudotumors (IPTs) are uncommon neoplasms of unknown etiology that have been reported in numerous anatomic sites. The occurrence of these tumors in the spleen is extremely rare with only sporadic case reports and small case series reported in the literature [1-4]. In contrast to those occurring in other anatomic sites, the IPTs that occur in the spleen and liver are typically associated with Epstein-Barr virus (EBV) [5-10] suggesting a distinctly different pathogenetic pathway in these locations. In addition, prior studies report that the spindle cell proliferation in the majority of splenic IPTs, are at least focally composed of follicular dendritic cell (FDC) proliferations. Based on the latter observation, some authors have suggested that splenic IPTs to be designated as inflammatory pseudotumor-like follicular dendritic cell tumors (FDCT) [7]. Furthermore, the documented lowmalignant potential with recurrences separates these tumors from the typical IPTs in other locations as well as from the more aggressive intra-abdominal conventional FDCTs.

In this study, we report two additional cases of EBVassociated IPTs involving the spleen. Despite histomorphological similarity, the nature of the spindle cell proliferations and the degree of EBV expression appeared distinctly different in these two neoplasms. One case demonstrated significant follicular dendritic cell proliferations documented by the follicular dendritic cell markers and abundant EBV mRNA expression using EBV-encoded RNA probes (EBER) whereas in the second case, the FDC proliferation appeared negligible to absent and EBV expression was present only focally.

Our findings suggest that splenic IPTs may represent a spectrum of tumors ranging from typical EBV-associated follicular dendritic cell proliferations to those resembling 
conventional IPTs with only focal EBV expression and minimal to absent FDC proliferations.

\section{Case reports}

Case 1

A 37-year-old male presented with a 4-month history of left-sided abdominal and flank pain, $8 \mathrm{lb}$ weight loss over a month, and frequent night sweats over the past 2 years. CT scan of the abdomen and pelvis revealed a $6-\mathrm{cm}$ solitary splenic mass (Fig. 1). There was no intra-abdominal or retroperitoneal lymphadenopathy. A splenectomy was performed and submitted for routine pathologic examination. Seventeen months after splenectomy, the patient remains asymptomatic.

\section{Case 2}

A 60-year-old female presented with abdominal and flank pain. The imaging studies revealed kidney stones and an incidental $5.5 \mathrm{~cm}$ in diameter solitary splenic mass. A splenectomy was performed and submitted for routine histologic evaluation. Nine months after splenectomy, the patient remains disease-free with no evidence of recurrence.

\section{Materials and methods}

Splenectomy specimens from each case were thinly sliced and fixed overnight in $10 \%$ buffered formalin, processed routinely, and representative sections were stained with hematoxylin and eosin for light microscopic examination. Portions of fresh splenic tissue on case 1 were submitted for

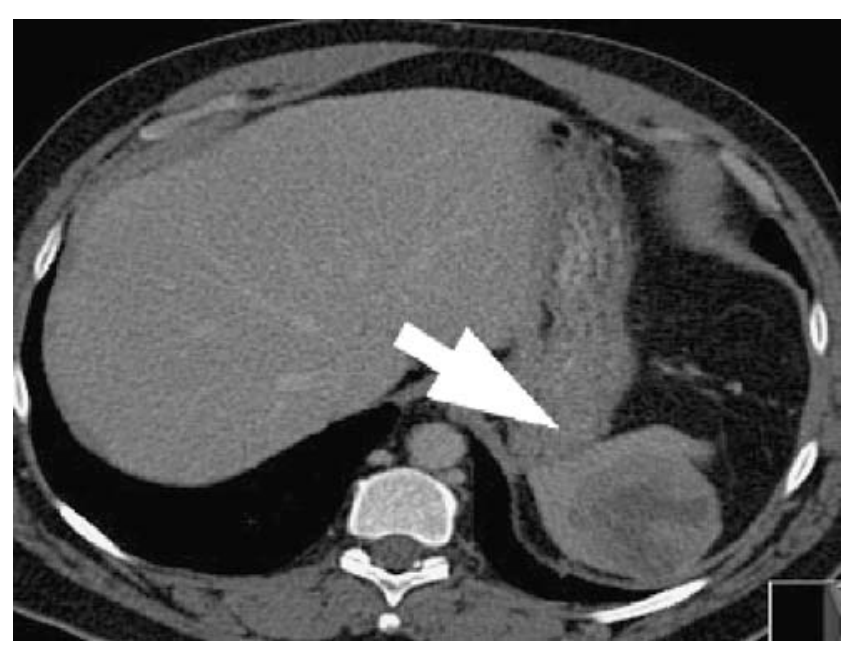

Fig. $1 \mathrm{CT}$ scan of the abdomen in case 1 demonstrates a solitary $6 \mathrm{~cm}$ in greatest dimension solid appearing splenic mass flow cytometric immunophenotyping and conventional cytogenetics.

Immunohistochemical studies

Immunohistochemical studies were performed using formalin fixed, paraffin-embedded tissue sections. The following antibodies were used: CD3, CD5, CD15, CD23, CD34, ALK protein (Ventana Medical Systems, Tucson, AZ, USA); smooth muscle actin (SMA), CD20, CD21, CD30, epithelial membrane antigen (EMA) (Dako, Carpinteria, CA, USA); CD35 (Cell Marque, Rocklin, CA, USA); BCL2 (Covance, Dedham, MA, USA); S-100 (BioCare, Concord, CA, USA); and HHV8 (Advanced Biotech, Rivers Park, IL, USA). The following antibodies were diluted using antibody dilution buffer (Ventana): CD3, CD5, CD15, CD23, CD34, CD35, ALK protein, and EMA at a dilution of 1:10; CD30 and BCL2 at 1:20; CD21 at 1:50; SMA at 1:100; CD20 at 1:200; S-100 at 1:800; and HHV8 at 1:1,000. Protease digestion (Ventana) was used for epitope retrieval for CD21 antibody. Heat-induced epitope retrieval was accomplished in a microwave oven for the remaining antibodies using citrate buffer on an XT Benchmark Instrument (Ventana). Reactivity was detected using the Ultra-View detection kit (Ventana).

In situ hybridizations for EBV and light chains

In situ hybridization for Epstein-Barr virus were performed using a cocktail of EBV-encoded small RNA probes labeled with fluorescein conjugate in a formamide-based diluent (Inform EBER, Ventana). The intended target is the early RNA transcripts of EBV accumulated in the nucleus of EBV-infected cells. The probes are designed specifically for the BenchMark Automated Staining System (Ventana). A known EBV-infected tissue section served as a positive control and EBV-negative lymphoid tissue served as a negative control in each run.

In situ hybridization for immunoglobulin kappa and lambda light chains were performed using a cocktail of oligonucleotide probes labeled with a fluorescein conjugate in a formamide-based diluent (Inform Cytoplasmic Immunoglobulin $\mathrm{K}$ and L, Ventana). The intended target is cytoplasmic immunoglobulin kappa or lambda light chains mRNA. Sections of a benign tonsil were used as quality controls.

\section{Results}

The splenectomy specimens weighed 237 and $167 \mathrm{~g}$, respectively. Each spleen contained a well-circumscribed tan fleshy mass with small necrotic appearing foci. The 


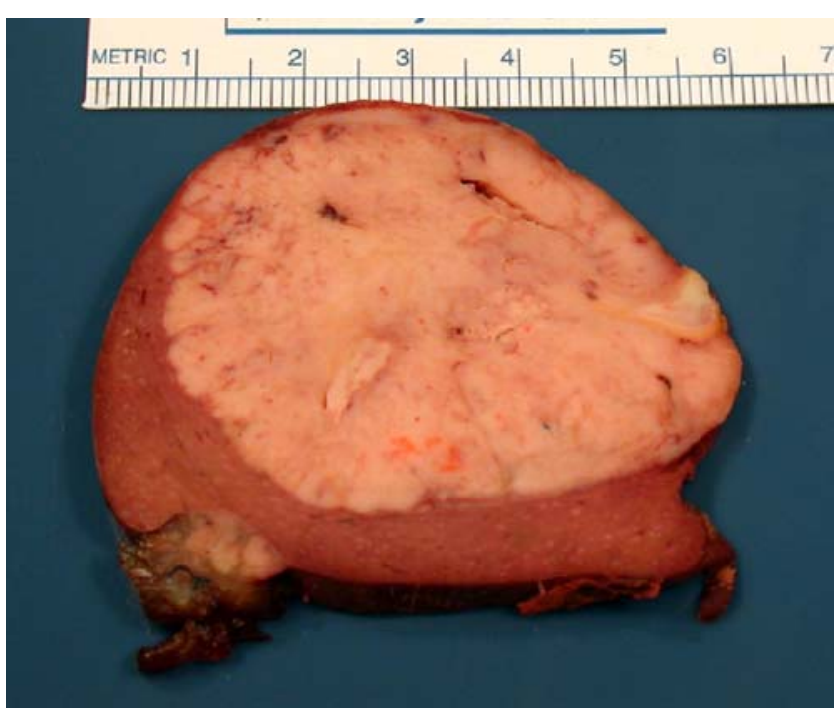

Fig. 2 Cut surface of the splenectomy specimen in case 1 demonstrates a well-circumscribed $6 \mathrm{~cm}$ in diameter fleshy mass with small foci of hemorrhage and necrosis

masses measured $6 \mathrm{~cm}$ (Fig. 2) and $5.5 \mathrm{~cm}$ in greatest dimension, respectively.

Histologic, immunohistochemical, EBER, flow cytometric, and cytogenetic findings

\section{Case 1}

Histologic examination of hematoxylin and eosin-stained sections of the splenic mass in case 1 revealed a mixed inflammatory infiltrate with abundant mature plasma cells and a proliferation of spindled cells with foci showing a storiform pattern and abundant large ovoid cells with

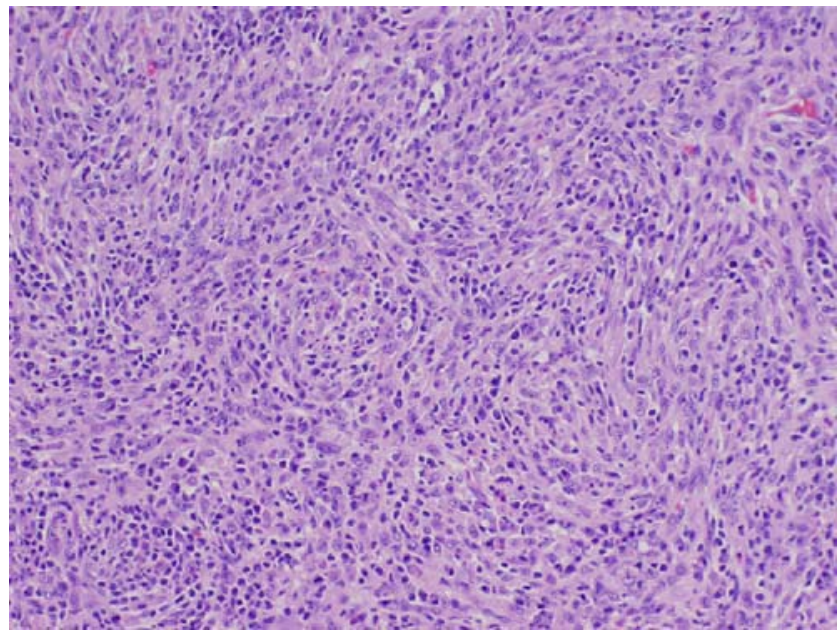

Fig. 3 Histologic section of splenic tumor in case 1 shows a mixed inflammatory infiltrate and a proliferation of spindle cells with storiform pattern. Hematoxylin and eosin stain, original magnification, $\times 200$

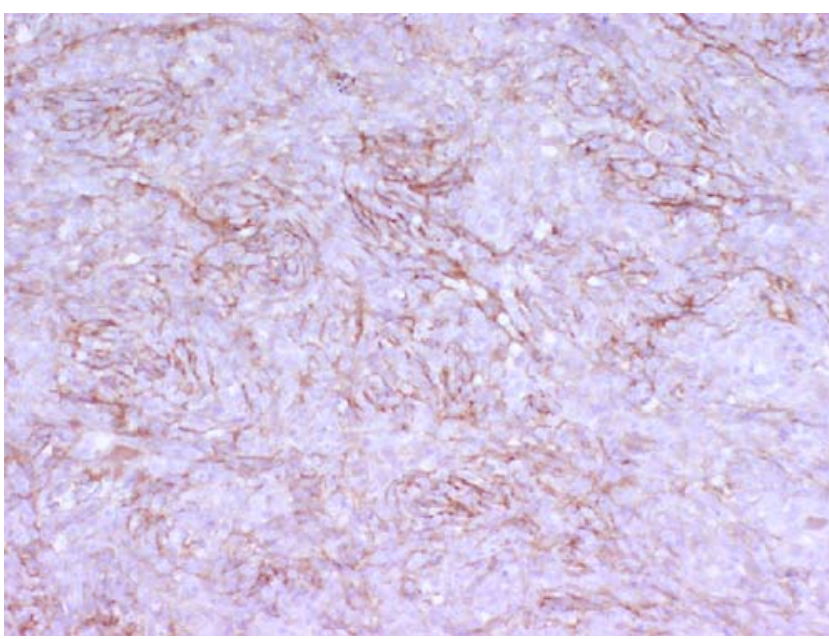

Fig. 4 Immunohistochemical stain using anti-CD35 antibody in case 1 highlights foci of follicular dendritic cell proliferation. Immunoperoxidase stain, original magnification, $\times 200$

vesicular nuclei and small nucleoli (Fig. 3). No overt malignant appearing cells were identified. In several foci, the oval and spindle cells were positive for the follicular dendritic cell markers including CD21, CD23, and CD35 (Fig. 4). In situ hybridization for EBV (EBER) showed abundant EBV-infected cells that included the proliferating oval and spindle cells (Fig. 5). The oval cells were negative for CD15, CD30, ALK protein, S-100, EMA, CD34, and HHV-8. Stains for CD20 and CD3 highlighted the background $\mathrm{B}-$ and T-cells, with a slight predominance of CD20-positive B-cells. No aberrant co-expression of CD5 or BCL2 was identified on B-cells. In situ hybridization for immunoglobulin kappa and lambda light chains revealed polytypic plasma cells. Flow cytometric immunophenotyping revealed no monoclonal B-cell or abnormal T-cell

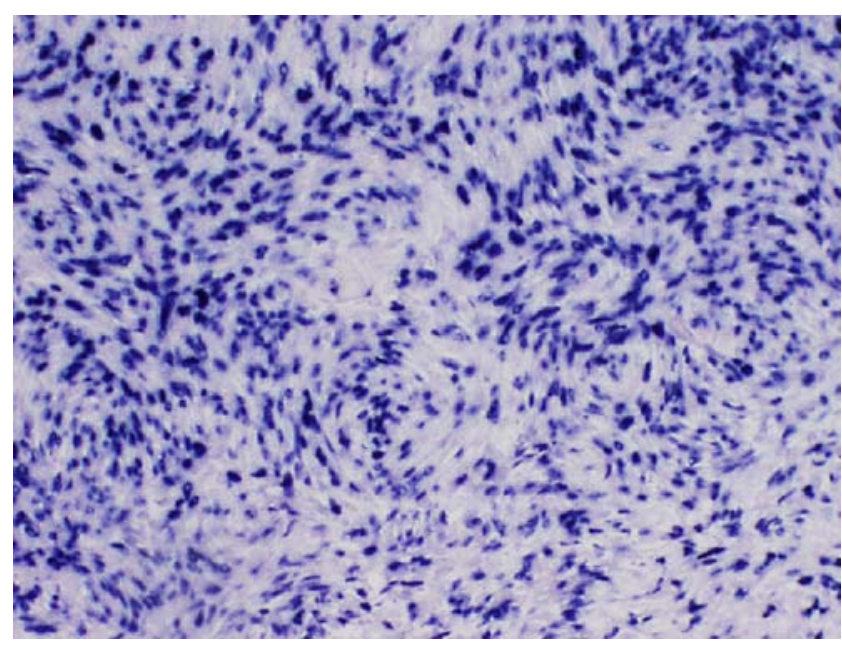

Fig. 5 In situ hybridization using EBV-encoded small RNA probes demonstrates numerous EBV-infected cells including oval and spindle cells in case 1 . Original magnification, $\times 200$ 


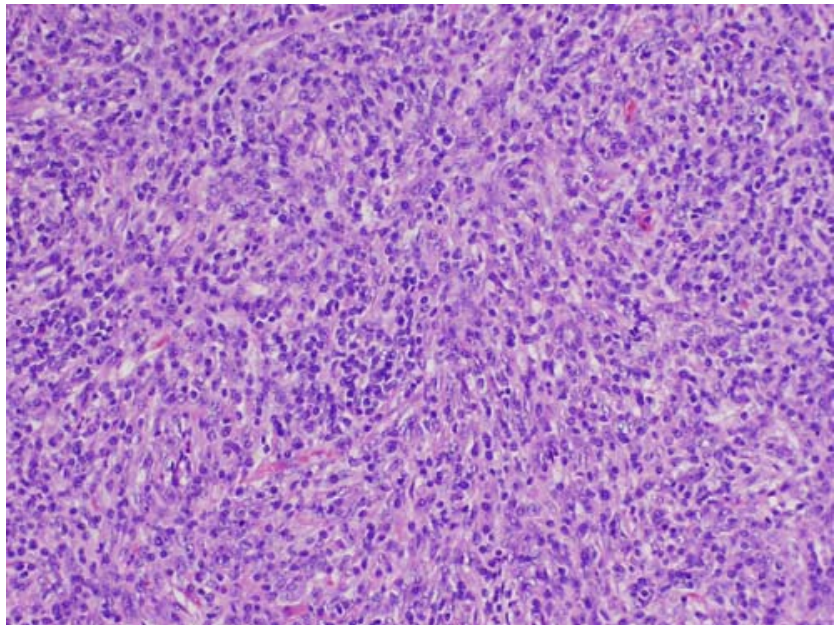

Fig. 6 Histologic section of splenic mass in case 2 shows a mixed infiltrate including many mature appearing plasma cells and occasional oval and spindle cells. Hematoxylin and eosin stain, original magnification, $\times 200$

population. Conventional cytogenetics revealed normal male karyotype with no numerical or structural abnormality.

\section{Case 2}

Microscopic examination of hematoxylin- and eosin-stained sections of splenic mass revealed histologic findings similar to those described for case 1 . The tumor showed a mixed inflammatory infiltrate with a proliferation of spindled cells focally in a storiform pattern (Fig. 6). The spindle cells were positive for SMA (Fig. 7). A very faint focal positivity was noted for CD35. However, the remaining follicular dendritic markers, CD21 and CD23 were negative. In situ hybridization for EBV (EBER) revealed focal weak EBV

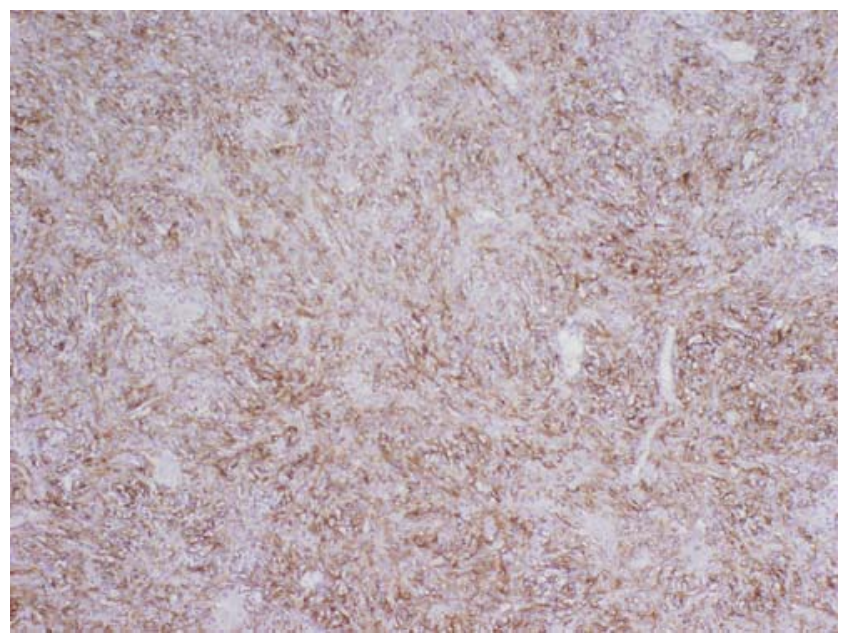

Fig. 7 Immunohistochemical stain using anti-smooth muscle actin (SMA) antibody in case 2 highlights most oval and spindle cells. Immunoperoxidase stain, original magnification $\times 200$

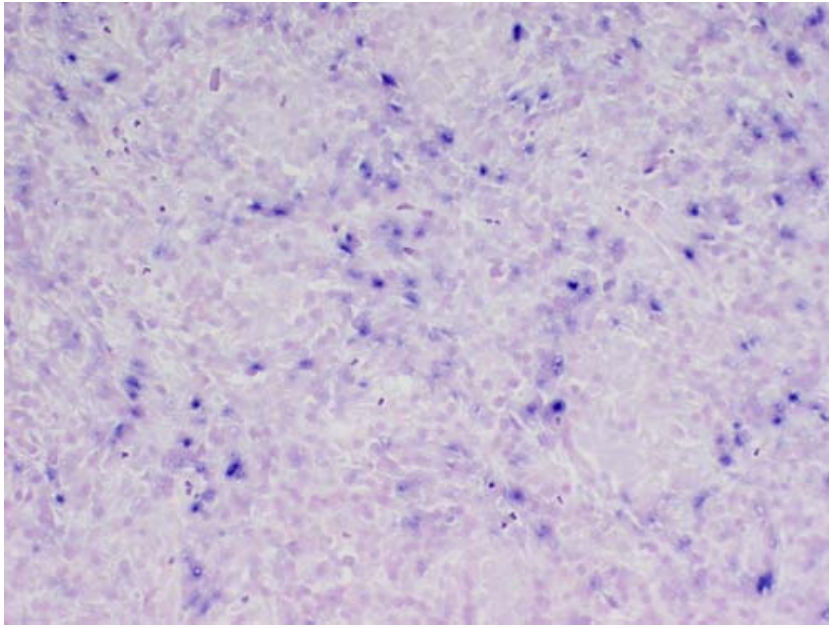

Fig. 8 In situ hybridization using EBV-encoded small RNA probes demonstrates focal weak EBV-positive cells in scattered oval to spindle cells in case 2 . Original magnification, $\times 200$

positivity in scattered oval and spindle cells (Fig. 8) correlating to SMA-positive cells. The spindle cells were negative for CD30, ALK protein, and BCL2. CD20 and CD3 highlighted the background B- and T-cells with no aberrant co-expression of CD5 or BCL2 by B-cells. In situ hybridization for immunoglobulin kappa and lambda light chains revealed polytypic plasma cells.

\section{Discussion}

Inflammatory pseudotumor (IPT) also known as inflammatory myofibroblastic tumor is an uncommon lesion of uncertain origin that occurs in numerous anatomic sites. The splenic presentation is rare with only sporadic case reports and small case series reported in the literature [1-4]. The previously reported and documented recurrences, in at least a subset of those occurring in the liver and spleen, support a neoplastic nature with a low-grade malignant potential in these two locations [7]. These observations clearly indicate that the IPT occurring in these two locations exhibit a distinctly different biologic behavior compared to those presenting in other locations with a clinical behavior suggestive of a non-neoplastic proliferation. The spindle cell proliferation in the majority of splenic and hepatic IPTs are at least focally composed of follicular dendritic cell proliferations that can be demonstrated using sensitive FDC-associated immunohistochemical markers. Based on this observation, some authors have suggested that IPTs that occur in spleen and liver should be designated as inflammatory pseudotumor-like follicular dendritic cell tumors [7]. However, several studies have shown that the spindle cells in significant proportions of IPT of the spleen are composed of myofibroblastic proliferations [1, 4]. The 
presence of myofibroblasts in IPT of spleen has led to designation of "inflammatory myofibroblastic (IMF) tumor" by some authors $[1,4]$. However, the IPTs of the spleen that have been classified as IMF tumors should not be confused with soft tissue IMF neoplasms that often harbor balanced translocations involving ALK kinase gene and exhibit a more aggressive clinical behavior [11].

In contrast to those in other locations, most IPTs in liver and spleen are EBV-associated [7-9]. Using in situ hybridization methods, the EBV is localized to spindle cell proliferations in the vast majority of the IPTs in spleen including those with absence of follicular dendritic cell markers [10]. In addition, the EBV in the IPT in the spleen and liver is clonal when assessed by Southern blot analysis of EBV DNA terminal repeat regions [10, 12, 13]. This finding provides additional evidence in support of a neoplastic nature for IPT occurring in liver and spleen.

The IPTs of the spleen with foci of FDC proliferations must be separated from conventional FDC tumors with intra-abdominal presentations that typically exhibit a more aggressive clinical behavior.

The majority of the patients with IPT of spleen present with B symptoms including weight loss, fever, and abdominal pain. On imaging, most cases of splenic IPTs present with solitary relatively large well-circumscribed masses. The clinical and radiologic findings raise the possibility of a lymphoma in most cases and the diagnosis of IPT in virtually all cases is made only after pathologic examination of splenectomy specimens in conjunction with ancillary studies.

On gross examination of splenectomy specimens, the tumors appear well-circumscribed with small foci of necrosis. Histologically, these tumors are composed of a mixture of lymphocytes, histiocytes, plasma cells, and a variable proportion of bland appearing spindle cells.

In this study, we report two additional cases of EBVassociated IPTs involving the spleen. Despite histomorphologic similarities, the nature of the spindle cell proliferations and the degree of EBV expression appeared distinctly different in these two neoplasms. Case 1 presented with weight loss, night sweats, and abdominal pain similar to those typically described in the prior studies in the literature. However, in case 2, the tumor was found incidentally at the time of work up for kidney stones. In addition, significant follicular dendritic cell proliferations documented by the follicular dendritic cell markers and abundant EBV mRNA expression using EBV-encoded small RNA probes were present in case 1; whereas in case 2, the FDC proliferation appeared negligible to absent and EBV expression was present only focally and in SMA-positive spindle cells. Seventeen months post-splenectomy, the first patient remains asymptomatic at last clinical follow-up with no evidence of recurrent tumor. Similarly, the second patient remains disease-free on last clinical follow-up 9 months post-splenectomy.

In summary, our findings suggest that splenic IPTs may represent a spectrum of proliferations ranging from typical IPT-like EBV-associated follicular dendritic cell proliferations to those resembling conventional IPTs with predominance of myofibroblastic proliferations and with minimal to absent FDC proliferations and only focal EBV expression. Additional studies with larger numbers of cases are needed to further define the nature of these rare EBVassociated proliferations in spleen.

\section{References}

1. Neuhauser TS, Derringer GA, Thompson LDR, Fanburg-Smith JC, Aguilera NSI, Andriko J, Chu W-S, Abbondanzo SL (2001) Splenic inflammatory myofibroblastic tumor (inflammatory pseudotumor). A clinicopathologic and immunophenotypic study of 12 cases. Arch Path Lab Med 125:79-85

2. Noguchi H, Kondo H, Kondo M, Shiraiwa M, Monobe Y (2000) Inflammatory pseudotumor of the spleen. A case report. Jpn J Clin Oncol 30:196-203

3. Chen WH, Liu TP, Liu CL, Tzen CY (2004) Inflammatory pseudotumor of the spleen. J Chin Med Assoc 67:533-536

4. Oshiro $\mathrm{H}$, Nomura $\mathrm{M}$, Yamanaka S, Watanabe S, Inayama $\mathrm{Y}$ (2007) Splenic inflammatory pseudotumor (inflammatory myofibroblastic tumor). J Clin Exp Hematopathol 47:83-88

5. Horiguchi H, Matsui-Horiguchi M, Sakta H, Ichinose M, Yamamoto T, Fujiwara M, Ohse H (2004) Inflammatory pseudotumor-like follicular dendritic cell tumor of the spleen. Pathol Inter 54:124-131

6. Oz Puyan F, Bilgi S, Unlu E, Yalcin O, Altaner S, Demir M, Cakir B (2004) Inflammatory pseudotumor of the spleen with EBV positivity. Report of a case. Eur J Haematol 72:285-291

7. Cheuk W, Chan JKC, Shek TWH et al (2001) Inflammatory pseudotumor-like follicular dendritic cell tumor. A distinctive low-grade malignant intra-abdominal neoplasm with consistent Epstein-Barr virus association. Am J Surg Pathol 25:721-731

8. Arber DA, Kamel OW, van de Rijn M, Davis RE, Medeiros LJ, Jaffe ES, Weiss LM (1995) Frequent presence of the EpsteinBarr virus in inflammatory pseudotumor. Hum Pathol 26:10931098

9. Arber DA, Weiss LM, Chang KL (1998) Detection of EpsteinBarr virus in inflammatory pseudotumor. Semin Diagn Pathol 15:155-160

10. Lewis JT, Gaffney RL, Casey MB, Farrell MA, Morice WG, Macon WR (2003) Inflammatory pseudotumor of the spleen associated with a clonal Epstein-Barr virus genome. Case report and review of the literature. Am J Clin Path 120:56-61

11. Kutok JL, Pinkus GS, Dorfman DM, Fletcher CDM (2001) Inflammatory pseudotumor of lymph node and spleen. An entity biologically distinct from inflammatory myofibroblastic tumor. Hum Pathol 32:1382-1387

12. Selves J, Meggetto F, Brousset $P$ et al (1996) Inflammatory pseudotumor of the liver. Evidence for follicular dendritic reticulum cell proliferation associated with clonal Epstein-Barr virus. Am J Surg Pathol 20:747-753

13. Shek TW, Ho FC, Ng IO et al (1996) Follicular dendritic cell tumor of the liver. Evidence for an Epstein-Barr virus-related clonal proliferation of follicular dendritic cells. Am J Surg Pathol 20:313-324 